\title{
Genetic polymorphism and natural selection of Duffy binding protein of Plasmodium vivax Myanmar isolates
}

Hye-Lim Ju ${ }^{1 \dagger}$, Jung-Mi Kang ${ }^{1 \dagger}$, Sung-Ung Moon ${ }^{2}$, Jung-Yeon Kim³ ${ }^{3}$, Hyeong-Woo Lee ${ }^{4}$, Khin Lin ${ }^{5}$, Woon-Mok Sohn ${ }^{1}$, Jin-Soo Lee ${ }^{6}$, Tong-Soo Kim ${ }^{7^{*}}$ and Byoung-Kuk Na ${ }^{1^{*}}$

\begin{abstract}
Background: Plasmodium vivax Duffy binding protein (PvDBP) plays an essential role in erythrocyte invasion and a potential asexual blood stage vaccine candidate antigen against $P$. vivax. The polymorphic nature of PvDBP, particularly amino terminal cysteine-rich region (PvDBPII), represents a major impediment to the successful design of a protective vaccine against vivax malaria. In this study, the genetic polymorphism and natural selection at PvDBPII among Myanmar P. vivax isolates were analysed.

Methods: Fifty-four P. vivax infected blood samples collected from patients in Myanmar were used. The region flanking PvDBPII was amplified by PCR, cloned into Escherichia coli, and sequenced. The polymorphic characters and natural selection of the region were analysed using the DnaSP and MEGA4 programs.

Results: Thirty-two point mutations (28 non-synonymous and four synonymous mutations) were identified in PvDBPII among the Myanmar P. vivax isolates. Sequence analyses revealed that 12 different PvDBPII haplotypes were identified in Myanmar P. vivax isolates and that the region has evolved under positive natural selection. High selective pressure preferentially acted on regions identified as B- and T-cell epitopes of PvDBPII. Recombination may also be played a role in the resulting genetic diversity of PvDBPII.

Conclusions: PvDBPII of Myanmar P. vivax isolates displays a high level of genetic polymorphism and is under selective pressure. Myanmar P. vivax isolates share distinct types of PvDBPII alleles that are different from those of other geographical areas. These results will be useful for understanding the nature of the $P$. vivax population in Myanmar and for development of PvDBPII-based vaccine.
\end{abstract}

Keywords: Plasmodium vivax, Duffy binding protein, Myanmar

\section{Background}

Plasmodium vivax Duffy binding protein (PvDBP) is one of the erythrocyte-binding proteins, which belongs to the large erythrocyte binding protein family [1]. PvDBP is expressed on the merozoite of $P$. vivax and plays an essential role in erythrocyte invasion of the parasite by mediating irreversible binding with its corresponding receptor, the duffy antigen receptor for chemokines

\footnotetext{
* Correspondence: tongsookim@inha.ac.kr; bkna@gnu.ac.kr

+ Contributed equally

'Department of Parasitology and Institute of Health Sciences, Gyeongsang

National University School of Medicine, Jinju 660-751, Korea

${ }^{7}$ Department of Parasitology and Inha Research Institute for Medical

Sciences, Inha University School of Medicine, Incheon 400-712, Korea

Full list of author information is available at the end of the article
}

(DARC), on the surface of erythrocytes [1-4]. Similar to other plasmodial proteins known to participate in such processes, PvDBP is suggested to be an important vaccine candidate antigen, because it elicits strong immune responses in humans $[5,6]$. Experimental evidences that antibodies against PvDBP inhibit the interaction of this protein with DARC in vitro and block the invasion of $P$. vivax into human erythrocytes also support the notion that this protein is a potential asexual blood stage vaccine candidate antigen against $P$. vivax [7-9].

PvDBP is divided into seven regions (regions I-VII), and the amino terminal cysteine-rich region, region II (PvDBPII), contains the central binding motifs necessary for adherence to DARC [10-12]. Critical binding motifs

\section{Biomed Central}


in PvDBPII have been mapped to a 170 amino acid stretch (amino acids 291-460), which includes cysteines 5-8 [11,12]. PvDBPII shows the highest genetic diversity compared to the remaining PvDBP regions and appears to be under strong selective pressure $[13,14]$. Analysis of genetic variation of PvDBPII among $P$. vivax field isolates from different geographical regions, including Brazil, Colombia, South Korea, Papua New Guinea, Thailand, showed that the PvDBPII is highly polymorphic, but the cysteine residues are conserved within and between $P$. vivax populations from different geographic regions [14-21]. Although it has been suggested that these polymorphisms do not significantly alter host-parasite binding $[17,22]$, some of them alter immune recognition of PvDBP [23] and most of the PvDBP-specific antibodies detected in infected individuals recognize PvDBPII, rather than other PvDBP regions $[7,15]$. Consequently, the polymorphic nature of PvDBP, particularly PvDBPII, represents a major impediment to the successful design of a protective vaccine against vivax malaria [14]. Therefore, understanding the nature and genetic polymorphism in PvDBPII among P. vivax isolates from distinct geographic areas, particularly where a large proportion of $P$. vivax infections occurs, is important for the rational design of vaccines against vivax malaria.

In this study, the genetic polymorphism and natural selection of PvDBPII among P. vivax isolates from Myanmar were analysed. These results suggest that excessive polymorphism of PvDBPII is found in the filed isolates of P. vivax in Myanmar.

\section{Methods}

\section{Blood samples and DNA preparation}

The 54 blood samples used in this study were collected from patients who were infected with Plasmodium vivax at Wet-Won Station Hospital, Pyin Oo Lwin township, Mandalay Division, Myanmar between 2004 and 2006 [24]. The confirmation of $P$. vivax infection was performed by microscopic examination of thin and thick blood smears and polymerase chain reaction [24]. Informed consent was obtained from all individuals participating in this study before blood sampling under the protocol approved by the Department of Health, The Union of Myanmar, and the Ethics Committee of the Centers for Disease Control and Prevention, Korea. Genomic DNA was extracted from $200 \mu \mathrm{l}$ of whole blood using a QIAamp Blood kit (Qiagen, Valencia, CA, USA), according to the manufacturer's instruction.

\section{Amplification and sequencing analysis of PvDBPII}

Amplification of the PvDBPII region was performed by the polymerase chain reaction (PCR) using the specific primers, PvDBPII F: 5'-ACCACGATCTCTAGTGCTATTATA-3' and PvDBPII R: 5'-ATTTGTCACAACTT
CCTGAGTATT-3'. The amplification reaction was performed using the following thermal cycling profile: $94^{\circ} \mathrm{C}$ for $5 \mathrm{~min}, 30$ cycles at $94^{\circ} \mathrm{C}$ for $1 \mathrm{~min}, 50^{\circ} \mathrm{C}$ for $1 \mathrm{~min}$, and $72^{\circ} \mathrm{C}$ for $1 \mathrm{~min}$, followed by a $72^{\circ} \mathrm{C}$ extension for 10 min. Ex Taq DNA polymerase (Takara, Otsu, Japan) was used in all PCR reactions to prevent any possible nucleotide mis-incorporation. The PCR product was analysed on a 1.2\% agarose gel, gel-purified, and ligated into the T\&A cloning vector (Real Biotech Cooperation, Banqiaa City, Taiwan). Each ligation mixture was transformed into Escherichia coli DH5 $\alpha$ competent cells, and positive clones were screened for the presence of the plasmid with the appropriate insert. The sequencing reaction was performed using the BigDye Terminator Cycle Sequencing Ready Reaction kit in an ABI 377 automatic DNA sequencer (Applied Biosystems, Foster City, CA, USA). To verify the sequences, sequence analysis was performed by analysing at least two clones from each isolate.

\section{Sequence and phylogenetic analyses}

Nucleotide and deduced amino acid sequences were analysed using the EditSeq program and Clustal in the Megalign program, a multiple alignment program of the DNASTAR package (DNASTAR, Madison, WI, USA). The phylogeny tree was constructed using the neighbourjoining method with MEGA4 version 4.0 [25]. Bootstrap proportions were used to assess the robustness of the tree with 1,000 bootstrap replications. The sequences reported here have been deposited in the GenBank database under the accession numbers JN255576-JN255587.

\section{DNA sequence polymorphism analysis}

DNA sequence polymorphism analysis was performed on 54 Myanmar PvDBPII sequences. The number of segregating sites (S), haplotype diversity ( $\mathrm{Hd}$ ), nucleotide diversity $(\pi)$, and average number of pairwise nucleotide differences within the population $(K)$ were calculated using the DnaSP ver. 5.10.00 package [26]. The $\pi$ was also calculated on a sliding window of 100 bases, with a step size of $25 \mathrm{bp}$ to estimate the stepwise diversity across PvDBPII. The rates of synonymous $(K \mathrm{~s})$ and non-synonymous $(K \mathrm{n})$ substitutions were estimated and compared by the Z-test $(\mathrm{P}<$ 0.05) in MEGA4 program [25] using the Nei and Gojobori's method [27] with the Jukes and Cantor correction. Tajima's D test [28] and Fu and Li's D and F tests using P. knowlesi PvDBPII as an outgroup [29] were performed on DnaSP 5.10.00 to test the neutral theory of evolution.

\section{Analysis of polymorphism associated with B- and T- cell epitopes}

To assess the possibility that diversity in PvDBPII within the Myanmar P. vivax isolates may have arisen from host's immune pressure, the genetic diversity in predicted or known B- and T-cell epitopes [14] and MHC binding 
regions [30] in PvDBPII was examined. Polymorphism of each region was analysed by DnaSP ver. 5.10.00 [26], as described above.

\section{Recombination parameters and linkage disequilibrium}

The recombination parameter $(\mathrm{R})$, which included the effective population size and probability of recombination between adjacent nucleotides per generation, and the minimum number of recombination events $(\mathrm{Rm})$ were measured using DnaSP ver. 5.10.00 [26]. Linkage disequilibrium (LD) between different polymorphic sites was computed in terms of the $\mathrm{R}^{2}$ index.

\section{Results}

\section{Genetic polymorphisms and amino acid changes}

The region corresponding to PvDBPII was amplified from the 54 Myanmar $P$. vivax isolates by PCR. Each amplified product was cloned into the T\&A cloning vector and sequenced in both directions. To verify the sequences, sequencing reactions were performed for at least two plasmid clones for each gene. No size polymorphism was found between the sequences. Analysis and comparison of the sequences against the Sal I (DQ156512) as a reference sequence at the nucleotide level showed that point mutations occurred at 32 positions among the Myanmar isolates. Twelve of these 32 mutations occurred at the first base of the codon, 10 at the second base and 10 at the third base of the codon, resulting in significant amino acid changes (28 non-synonymous and four synonymous mutations) across the PvDBPII among Myanmar isolates. Most of the non-synonymous polymorphisms were non-conservative, resulting in changes in the physico-chemical family of the respective amino acid. A sequence analysis of the deduced amino acid sequences classified them into 12 different haplotypes (haplotypes 1-12) with amino acid changes at 28 positions, in which 1 showed a trimorphic polymorphism (position 386) and the others were dimorphic (Figure 1A). Seventeen of the 28 changes were previously reported, whereas the remaining 11 changes (I310L, F344S, R391H, K455I, K473R, C477G, R490K, D528G, V533M, K541T, and A545V) were new changes that have not been reported previously. Haplotype 3 was predominant ( $n=16,29.6 \%)$ among the Myanmar isolates. High frequencies of variant amino acids (> 50\%), compared to the Sal I sequence, were found for L333F (27/54, $50.0 \%)$, D384G (46/54, 85.2\%), R390H (34/54, 63.0\%), L424I (45/54, 83.3\%), W437R (33/54, 61.1\%), and I503K $(42 / 54,77.7 \%)$ (Figure 1B). A BLAST search was conducted in the GenBank database to compare these haplotypes with the previously identified PvDBPII sequences. Comparison of the 12 haplotypes found in the Myanmar $P$. vivax isolates with the GenBank sequences revealed that 10 of them (except haplotypes 1 and 8) were novel. Phylogenetic analysis revealed that the 12 Myanmar haplotypes were widely distributed among different isolates from distinct geographic regions (Figure 2). Comparison of the most common variants in PvDBPII among presently studied $P$. vivax populations revealed that Myanmar isolates showed a pattern similar to Thailand isolates [20] but one that differed from Papua New Guinean, Colombian, Brazilian, Iranian, and Sri Lankan isolates [19,21,31] (Table 1). Although the Myanmar isolates showed similar amino acid changes compared to Thailand isolates, nine variants found in the Thailand isolates (R268S, S351C, I367T, S398T, T404R, Q433K, R436T, $\mathrm{N} 507 \mathrm{H}$, and T513K) were not identified in the Myanmar isolates. Meanwhile, 11 variations (I310L, F343S, R391H, K455I, K473R, C477G, R490K, D528G, V533M, K541T, and $\mathrm{A} 545 \mathrm{~V}$ ) found in the Myanmar isolates did not occur in the Thailand isolates.

\section{Nucleotide diversity and natural selection of PvDBPII}

DNA sequence analyses were conducted to determine nucleotide diversity and genetic differentiation at PvDBPII among the Myanmar P. vivax isolates. The average number of pairwise nucleotide differences $(K)$ for the $963 \mathrm{bp}$ of the PvDBPII region was 7.851 (Table $2)$. The overall haplotype diversity (Hd) and nucleotide diversity $(\pi)$ for all 54 sequences were $0.875 \pm 0.029$ and $0.0079 \pm 0.0004$, respectively (Table 2). Further analysis, with a sliding window plot (window length $100 \mathrm{bp}$, step size $25 \mathrm{bp}$ ) using the DnaSP package, revealed that $\pi$ diversity ranged from 0 to 0.027 . The two highest peaks of nucleotide diversity within the PvDBPII region were identified between nucleotide positions 370-580 (Figure $3 \mathrm{~A})$. To determine whether natural selection contributed to generation of this diversity in PvDBPII within the Myanmar $P$. vivax population, the rate of non-synonymous $(K \mathrm{n})$ to synonymous substitutions $(K \mathrm{~s})$ was estimated using the Nei and Gojobori's method [27], as implemented in the MEGA4 program [25]. The standard error was determined by 1,000 bootstrap replications. The rate of non-synonymous substitutions $(K \mathrm{n})$ $(0.00917)$ exceeded the rate of synonymous substitutions (Ks) (0.00278). The $K \mathrm{n} / \mathrm{Ks}$ ratio was 3.299 (Table 2). These results suggest that positive natural selection may be occurring in PvDBPII and favoring fixed amino acid replacement in certain areas of the protein. The Tajima's D statistics was 0.264 ( $\mathrm{P}>0.1)$, indicating a decrease in population size and/or balancing selection. The Fu and Li's D and F values were $1.867(\mathrm{P}<0.02)$ and $1.535(0.1>\mathrm{P}>0.05)$, respectively.

\section{Polymorphisms associated with B- and T-cell epitopes}

To evaluate the association between positive natural selection and host immune evasion, we examined the genetic diversity in predicted or known B-and T-cell epitopes [14] and MHC binding regions [30] in PvDBPII. Most 


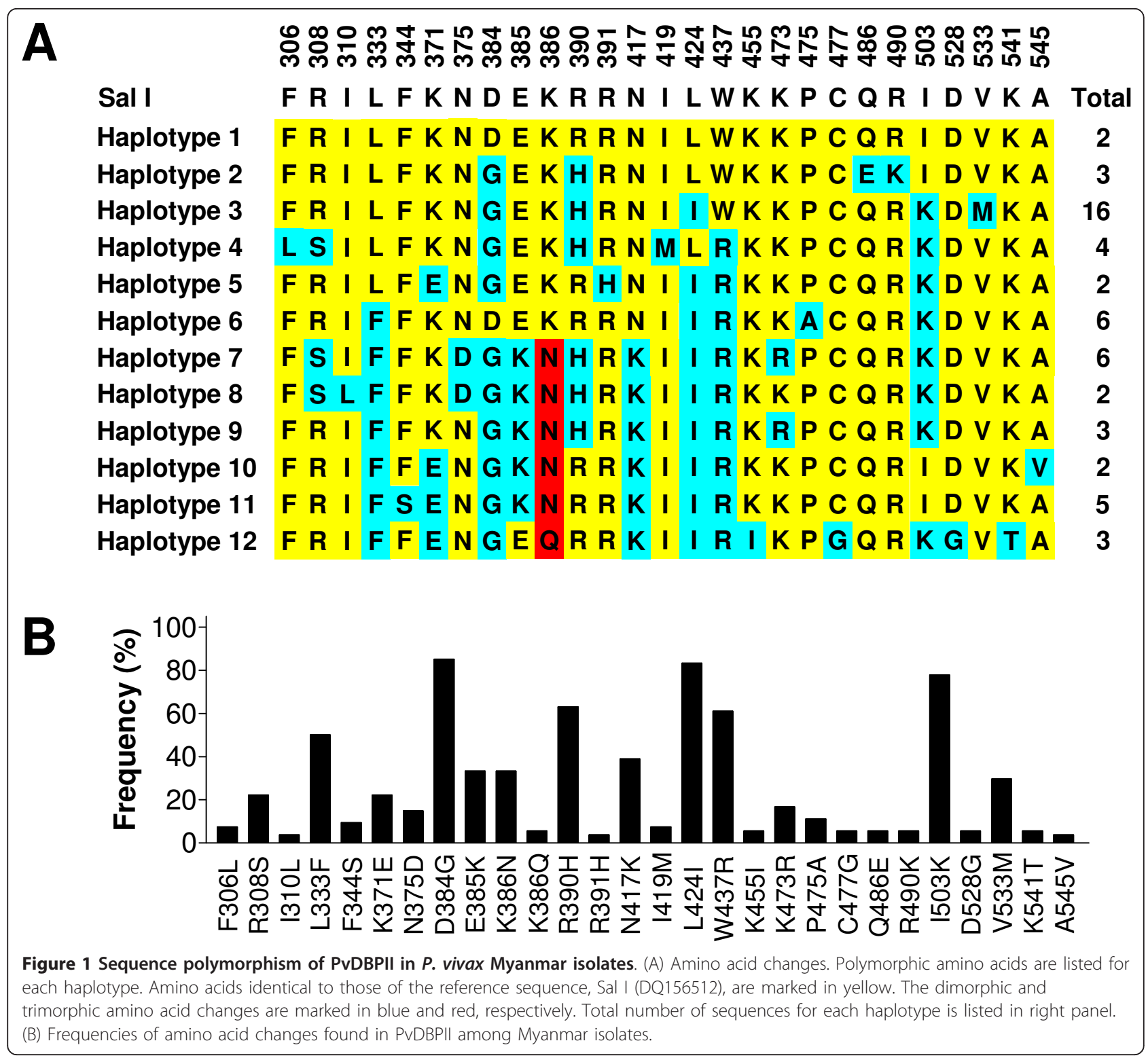

predicted $\mathrm{B}$ - and $\mathrm{T}$-cell epitopes and $\mathrm{MHC}$ binding regions were polymorphic, and most had a higher rate of non-synonymous mutations $(K n)$ than that of synonymous mutations $(K s)$ with the exceptions being the regions within peptides 13 and Ic (Table 3). High levels of nucleotide diversity were particularly found in peptides 48 and Ia, which contained spatially proximate polymorphic residues at positions 417, 419, and 427 and positions 385, 386, 390, and 391, respectively, and they showed a clear positive natural selection signature with positive Tajima's D values. In contrast, three peptides (28, 40 and 54), which did not contain known epitopes [14], had lower nucleotide diversity. These results are consistent with the hypothesis that natural selection acts on epitopes in PvDBPII and is related to diversity of PvDBPII [14,30].

\section{Recombination}

The minimum number of recombination events between adjacent polymorphic sites ( $\mathrm{Rm}$ ) was 7 , whereas $\mathrm{R}$ between adjacent sites $(\mathrm{Ra})$ and per gene $(\mathrm{Rb})$ was 0.0314 and 30.2, respectively. The high value of the recombination parameters indicated that high meiotic recombination may occur between sites, resulting in genetic diversity in the PvDBPII. The LD index $\mathrm{R}^{2}$ for the Myanmar population also declined across the analysed region, suggesting that intragenic recombination may also be contributing the increased diversity at PvDBPII (Figure 3B).

\section{Discussion}

Malaria is endemic or hypoendemic in Myanmar and is characterized by the occurrence of all four human- 


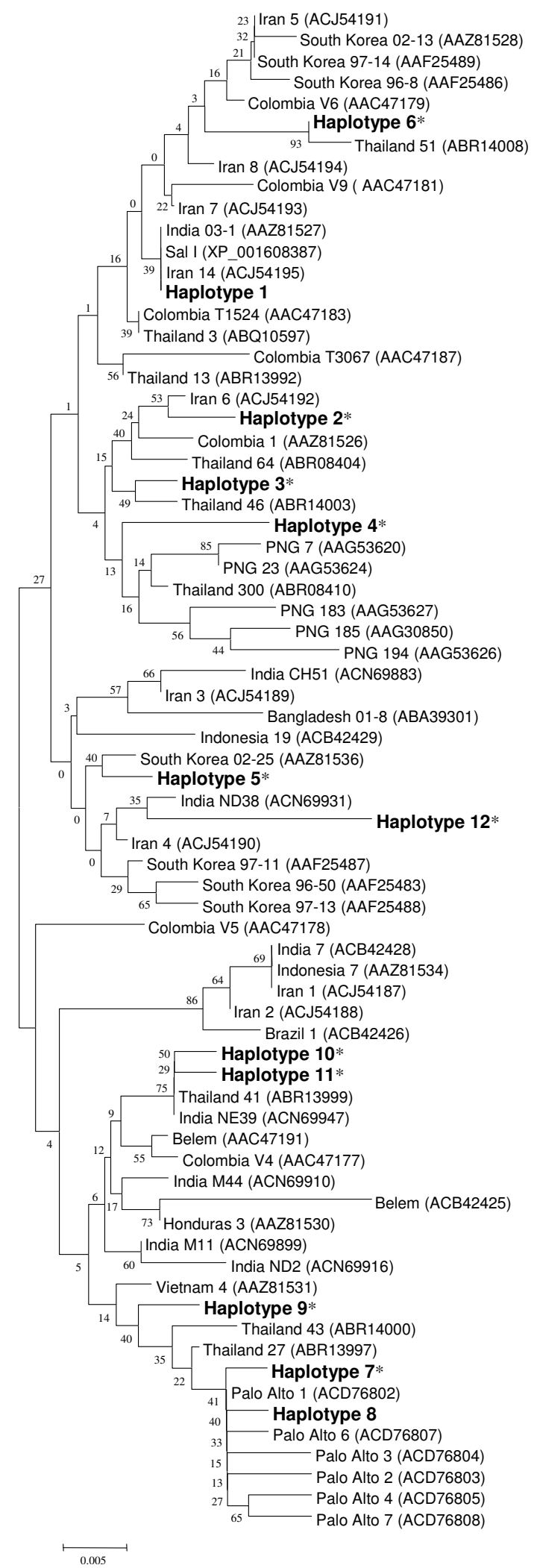

Figure 2 Phylogenetic analysis. The phylogenetic tree for the 12 haplotypes of PvDBPII was constructed with a neighbor-joining method using the MEGA4 program. Numbers on the branches indicate bootstrap proportions (1,000 replicates). Numbers on the branches indicate bootstrap proportions (1,000 replicates). The new haplotypes are marked with asterisks. 
Table 1 Frequencies of the most common variant amino acids in PvDBPII

\begin{tabular}{|c|c|c|c|c|c|c|c|c|c|c|}
\hline & F306L & R308S & D384G & $\mathrm{K} 386 \mathrm{~N}$ & K386Q & N417K & L424I & W437R & S447K & I503K \\
\hline Myanmar & 7.4 & 22.2 & 85.2 & 33.3 & 5.6 & 38.9 & 83.3 & 61.1 & 0 & 77.8 \\
\hline Thailand $^{\mathrm{a}}$ & 6.7 & 26.7 & 76.7 & 40.0 & 3.0 & 36.6 & 86.7 & 63.3 & 0 & 56.7 \\
\hline $\operatorname{Iran}^{\mathrm{b}}$ & 5.3 & 6.6 & 61.3 & 6.6 & 0 & 44.0 & 50.6 & 45.3 & 0 & 70.6 \\
\hline Sri Lanka ${ }^{c}$ & 7.0 & 13.0 & 94.0 & 20.0 & 0 & 36.0 & 49.0 & 37.0 & 0 & 55.0 \\
\hline Papua New Guinea $^{d}$ & 0 & 67.0 & 66.0 & 8.0 & 11.0 & 23.0 & 34.0 & 26.0 & 59.0 & 29.0 \\
\hline Colombia $^{d}$ & 0 & 0 & 59.0 & 23.0 & 0 & 47.0 & 47.0 & 18.0 & 0 & 12.0 \\
\hline Brazil $^{d}$ & 0 & 12.5 & 85.0 & 12.5 & 0 & 27.5 & 32.5 & 27.5 & 0 & 55.0 \\
\hline
\end{tabular}

${ }^{\mathrm{a}}[20] i^{\mathrm{b}}[21] ;{ }^{\mathrm{c}}[31] ;{ }^{\mathrm{d}}[19]$

The first letter represents the amino acid in that position in Sal I sequence, and the other letter represents the substituted amino acid

infecting Plasmodium species [32]. Although morbidity and mortality rates due to malaria have been declining gradually in recent years, Myanmar still contributes to approximately $60 \%$ of malaria deaths in the Southeast Asia [32]. Genetic polymorphisms in the circumsporozoite protein, merozoite surface protein-1 (MSP-1), MSP-3 $\alpha$, and apical membrane antigen-1 (AMA-1) of P. vivax Myanmar isolates were analysed previously $[24,33,34]$. As expected, they showed high levels of genetic polymorphisms, but information on the nature and extent of population diversity within malaria parasites in Myanmar is still limited. In this study, genetic polymorphism and natural selection of PvDBPII in Myanmar $P$. vivax isolates were analysed to expand our knowledge on population diversity of the parasite in the country.

A total of 54 PvDBPII sequences were obtained from Myanmar $P$. vivax isolates. The sequence analysis revealed that the 54 sequences were classified into 12 different haplotypes. A GenBank search for each haplotype revealed that two haplotypes were identical to at least one previously reported PvDBPII sequence found in other regions of the world, whereas the other 10 haplotypes were novel and have not been reported so far. The sequence analysis revealed that a total of 32 point mutations was identified, which resulted in significant amino acid changes (28 nonsynonymous and four synonymous changes) through the PvDBPII in Myanmar isolates. Seventeen of the 28 nonsynonymous changes were previously reported, whereas the other 11 changes (I310L, F344S, R391H, K455I, K473R, C477G, R490K, D528G, V533M, K541T, and A545V) were unique to Myanmar isolates, which did not hitherto identified. The two highest peaks of nucleotide diversity within PvDBPII in the Myanmar P. vivax isolates were identified between $\mathrm{C} 5$ and $\mathrm{C} 7$, which was consistent with previous observations $[14,15,17]$. Interestingly, a unique amino acid change (C477G caused by TGT to GGT) was identified in three Myanmar isolates. It is well known that the cysteine residues within PvDBPII are well conserved within and between $P$. vivax populations from different geographic regions studies [14-21,31]. It is not certain the nature of this amino acid change. It can be assumed that this mutation may resulted from a PCR or sequencing error, but to eliminate any possible errors, high quality Taq polymerase with a proof-reading function was used in all amplification processes and sequencing reactions were performed on at least two individual clones for each gene in both directions. But, considering the small number of isolates used in this study, further analyses with a large number of isolates is necessary to confirm this change in the Myanmar $P$. vivax isolates. Taken together, the results of this study suggest that PvDBPII in the Myanmar isolates showed a high level of genetic polymorphism with a nucleotide diversity $(\pi)$ of $0.0079 \pm$ 0.0004 .

Some polymorphic residues in PvDBPII occurred in only one population or geographic region, but common variant amino acids (K371E, D384G, E385K, K386N, N417K, L424I, W437R and I503K) are found in global isolates [19-21,31]. A high frequency (> 50\%) of D384G (85.2\%), R390H (63.0\%), L424I (83.3\%), W437R (61.1\%), and I503K (77.7\%) residues were found in Myanmar isolates compared to that in the Sal I sequence. This was highly similar to a report on Thailand isolates (D384G, 76.7\%; R390H,

Table 2 Estimates of DNA sequence polymorphism and tests of neutrality at PvDBPII among Myanmar isolates

\begin{tabular}{|c|c|c|c|c|c|c|c|c|c|c|c|}
\hline $\begin{array}{c}\text { Total } \\
\text { no. } \\
\text { of } \\
\text { isolates }\end{array}$ & $\begin{array}{l}\text { Segregating } \\
\text { sites (S) }\end{array}$ & $\begin{array}{c}\text { Singleton } \\
\text { variable sites }\end{array}$ & $\begin{array}{c}\text { Parsimony } \\
\text { informative sites }\end{array}$ & $\begin{array}{c}\text { Total no. } \\
\text { of } \\
\text { mutations }\end{array}$ & $K$ & $\mathrm{H}$ & $\mathrm{Hd} \pm \mathrm{SD}$ & $\pi \pm S D$ & $K \mathrm{n}$ & Ks & Tajima's \\
\hline 54 & 32 & 0 & 32 & 32 & 7.581 & 12 & $\begin{array}{c}0.875 \pm \\
0.029\end{array}$ & $\begin{array}{c}0.0079 \pm \\
0.0004\end{array}$ & 0.00917 & 0.00278 & $\begin{array}{c}0.264(P> \\
0.1)\end{array}$ \\
\hline
\end{tabular}

$\bar{K}$, average number of pairwise nucleotide differences; $\mathrm{H}$, number of haplotypes; $\mathrm{Hd}$, haplotype diversity; $\pi$, observed average pairwise nucleotid diversity; $K \mathrm{n}$, rate of non-synonymous mutations; Ks, rate of synonymous mutations 


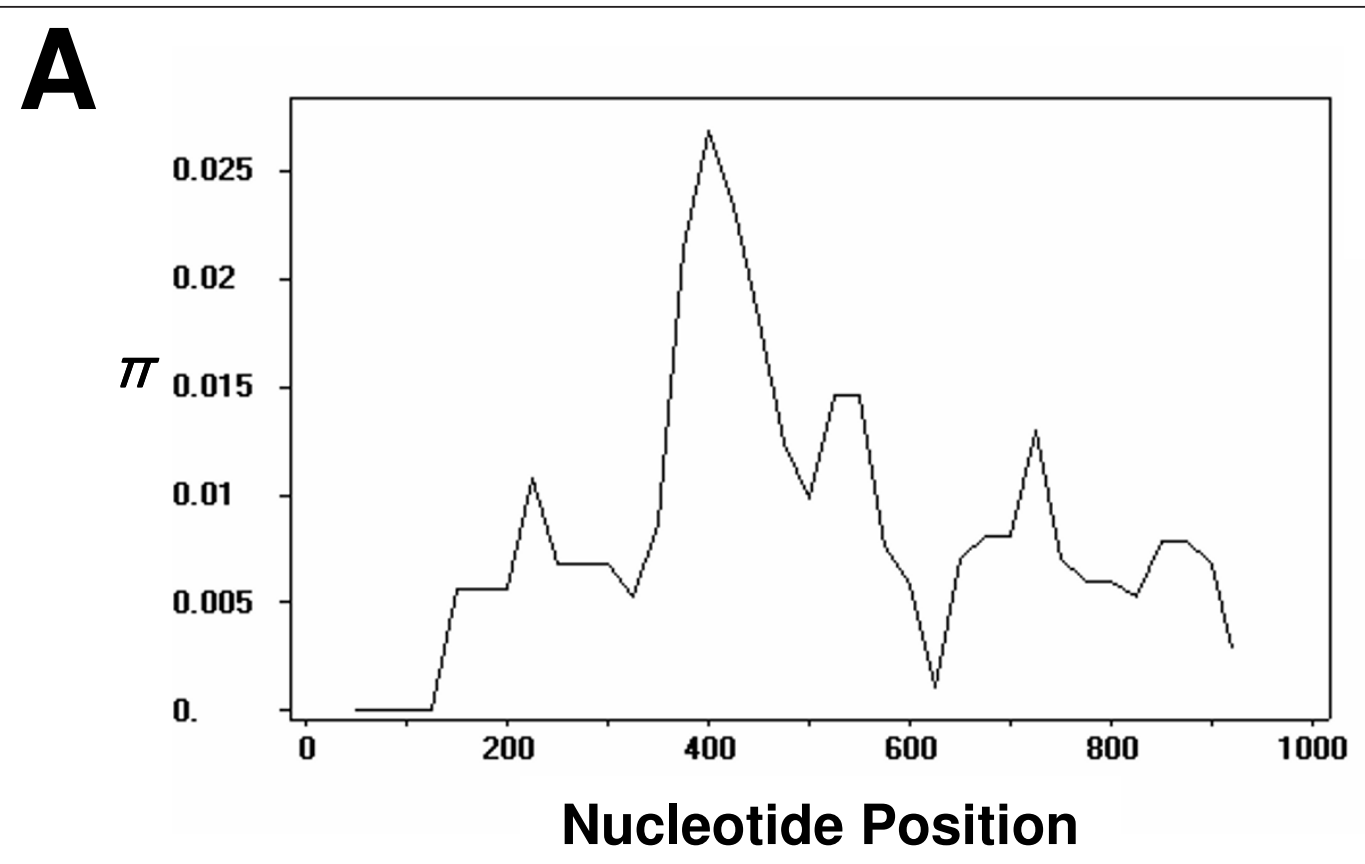

B

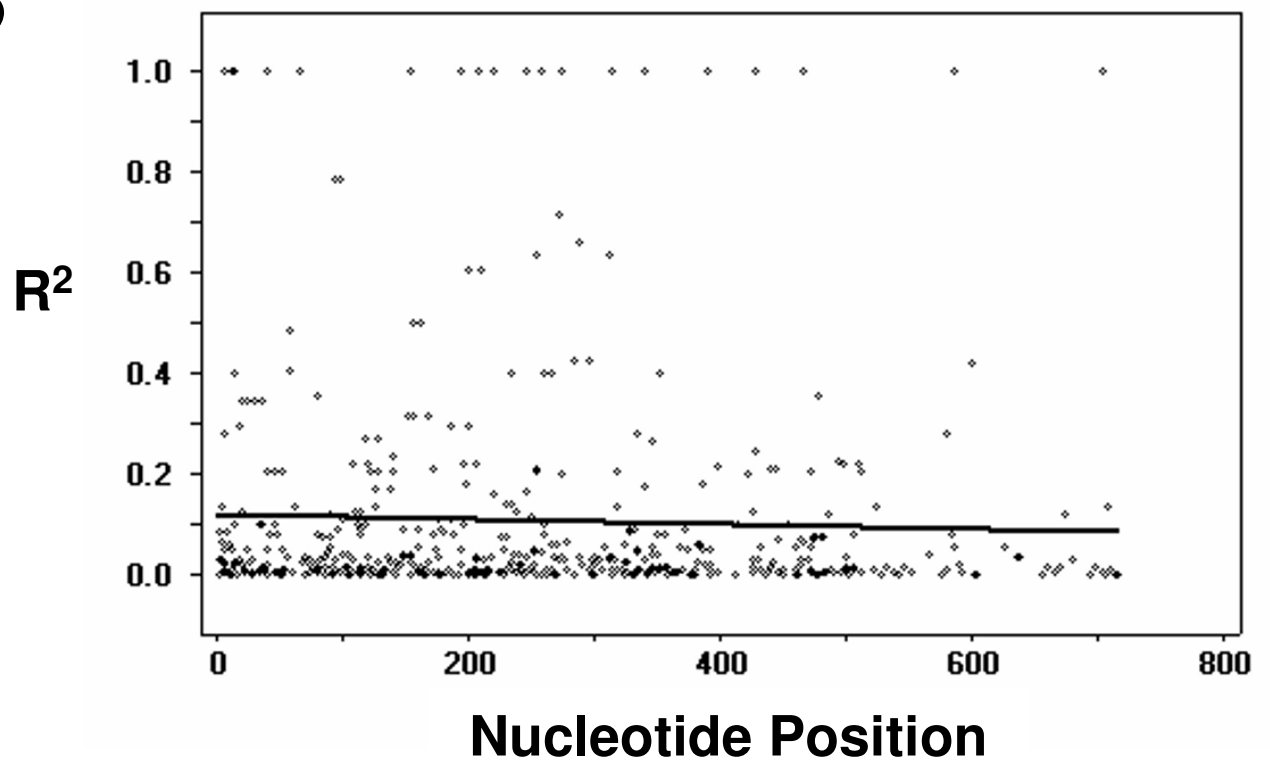

Figure 3 Natural selection of PvDBPII. (A) Sliding window plot of nucleotide diversity per site ( $\pi$ ) comparing the level of genetic diversity at PvDBPII. The $\pi$ values were calculated on DnaSP with window length $100 \mathrm{bp}$ and step size of $25 \mathrm{bp}$. (B) The linkage disequilibrium (LD) plot showing non-random association between nucleotide variants in 54 Myanmar P. vivax isolates at different polymorphic sites. The $R^{2}$ values are plotted against the nucleotide distances with two-tailed Fisher's exact test of significance.

56.7\%; L424I, 86.7\%; W437R, 63.3\%; I503K, 56.7\%) [20] but differed from previous studies showing R308S (67\%), D384G (66\%), and S447K (59\%) in Papua New Guinean isolates; D384G (59\%) in Colombian isolates; D384G
(85\%) and I503K (55\%) in Brazilian isolates; D384G $(61.3 \%)$ and I503K (70.6\%) in Iranian isolates; and D384G (94\%) and I503K (55\%) in Sri Lankan isolates [19,21,31]. F306L, which has only been reported from Asian malaria 
Table 3 Polymorphism observed in the each epitope sequence

\begin{tabular}{|c|c|c|c|c|c|c|c|c|c|c|c|c|}
\hline Epitope name & Epitop $^{a}$ & Segregating sites (S) & $\begin{array}{l}\text { Singleto } \\
\text { n variable } \\
\text { sites }\end{array}$ & $\begin{array}{c}\text { Parsimony } \\
\text { informative sites }\end{array}$ & $\begin{array}{c}\text { Total no. } \\
\text { of mutations }\end{array}$ & K & $\mathrm{H}$ & $\mathrm{Hd} \pm \mathrm{SD}$ & $\pi \pm S D$ & $K \mathrm{n}$ & Ks & Tajima's D \\
\hline 5 & $\mathrm{~T} / \mathrm{B}$ & 3 & 0 & 3 & 3 & 0.565 & 4 & $0.383 \pm 0.079$ & $0.013 \pm 0.003$ & 0.016 & 0 & $-0.289(P>0.1)$ \\
\hline 13 & $\mathrm{~T}$ & 0 & 0 & 0 & 0 & 0 & 1 & 0 & 0 & 0 & 0 & 0 \\
\hline 16 & $\mathrm{~T} / \mathrm{B}$ & 1 & 0 & 1 & 1 & 0.509 & 2 & $0.509 \pm 0.013$ & $0.011 \pm 0.000$ & 0.015 & 0 & $1.741(P>0.05)$ \\
\hline 18 & B & 1 & 0 & 1 & 1 & 0.509 & 2 & $0.509 \pm 0.013$ & $0.011 \pm 0.000$ & 0.015 & 0 & $1.741(P>0.05)$ \\
\hline 20 & $\mathrm{~T} / \mathrm{B}$ & 1 & 0 & 1 & 1 & 0.509 & 2 & $0.509 \pm 0.013$ & $0.011 \pm 0.000$ & 0.014 & 0 & $1.741(P>0.05)$ \\
\hline 28 & None & 0 & 0 & 0 & 0 & 0 & 1 & 0 & 0 & 0 & 0 & 0 \\
\hline 40 & None & 3 & 0 & 3 & 3 & 0.867 & 3 & $0.542 \pm 0.059$ & $0.019 \pm 0.003$ & 0.018 & 0.025 & $0.642(P>0.1)$ \\
\hline 48 & B & 5 & 0 & 5 & 5 & 1.560 & 6 & $0.748 \pm 0.040$ & $0.035 \pm 0.003$ & 0.041 & 0 & $1.007(P>0.1)$ \\
\hline 54 & None & 0 & 0 & 0 & 0 & 0 & 1 & 0 & 0 & 0 & 0 & 0 \\
\hline 66 & $\mathrm{~T}$ & 1 & 0 & 1 & 1 & 0.283 & 2 & $0.283 \pm 0.068$ & $0.006 \pm 0.002$ & 0.008 & 0 & $0.382(P>0.1)$ \\
\hline 78 & B & 1 & 0 & 1 & 1 & 0.107 & 2 & $0.107 \pm 0.055$ & $0.002 \pm 0.001$ & 0.003 & 0 & $-0.675(P>0.1)$ \\
\hline la & $\mathrm{MHCla}$ & 3 & 0 & 3 & 3 & 0.907 & 4 & $0.649 \pm 0.037$ & $0.034 \pm 0.004$ & 0.040 & 0 & $0.767(P>0.1)$ \\
\hline $\mathrm{Ib}$ & $\mathrm{MHClb}$ & 2 & 0 & 2 & 2 & 0.214 & 2 & $0.107 \pm 0.055$ & $0.008 \pm 0.004$ & 0.010 & 0 & $-0.898(P>0.1)$ \\
\hline IC & $\mathrm{MHClc}$ & 2 & 0 & 2 & 2 & 0.492 & 3 & $0.458 \pm 0.066$ & $0.012 \pm 0.002$ & 0.011 & 0.017 & $0.212(P>0.1)$ \\
\hline Ila & MHClla & 2 & 0 & 2 & 2 & 0.557 & 3 & $0.511 \pm 0.043$ & $0.019 \pm 0.002$ & 0.021 & 0.011 & $0.471(P>0.1)$ \\
\hline$\| \mathrm{b}$ & MHCllb & 2 & 0 & 2 & 2 & 0.423 & 3 & $0.297 \pm 0.076$ & $0.016 \pm 0.004$ & 0.019 & 0 & $-0.064(P>0.1)$ \\
\hline
\end{tabular}

$K$, average number of pairwise nucleotide differences; $\mathrm{H}$, number of haplotypes; $\mathrm{Hd}$, haplotype diversity; $\pi$, observed average pairwise nucleotide diversity; $K \mathrm{n}$, rate of non-synonymous mutations; $K \mathrm{~s}$, rate of synonymous mutations. ${ }^{a}$ B, B-cell epitope; T, T-cell epitope; none, no epitope identified 
endemic areas, including Thailand [20], Iran [21], and Sri Lanka [31], was also identified in the Myanmar isolates. Although the Myanmar isolates showed similar amino acid changes compared to those in the Thailand isolates, 9 variants found in the Thailand isolates (R268S, S351C, I367T, S398T, T404R, Q433K, R436T, N507H, and T513K) were not identified in the Myanmar isolates. Meanwhile, 11 variations (I310L, F344S, R391H, K455I, K473R, C477G, R490K, D528G, V533M, K541T, and A545V) found in the Myanmar isolates did not occur in the Thailand isolates. These results indicate that the Myanmar isolates are different from the Thailand isolates even though the two countries are very close geographically.

Although polymorphic residues were widely distributed throughout the PvDBPII sequence, polymorphisms at residues 417,437 , and 503 , either in single or in combination, can affect the efficacy of inhibitory antibodies against erythrocyte binding [23,35]. As these residues compose an important discontinuous epitope in PvDBP, which might be the main target for inhibitory antibodies, these polymorphisms could be subject to immune pressure responsible for parasite escape from the host immune system. It has been confirmed that this strong positive selection pressure in PvDBPII promotes greater diversity $[14,30]$. The immune pressure drives the generation of new PvDBP variants that are still able to bind erythrocytes but become resistant to inhibitory antibodies, suggesting that this DBP region is under positive pressure at critical residues and under negative pressure at the residues involved in receptor recognition [22,23,35]. A low prevalence of variant N417K (38.9\%) was observed among Myanmar isolates, but more than 50\% of W437R (61.1\%) and I503K $(77.8 \%)$ were identified. Analyses of the combination of variants revealed that W437R-I503K occurred at a higher frequency (70.4\%), whereas N417K-W437K and N417KI503K occurred at frequencies of $38.9 \%$ and $25.9 \%$, respectively. This result suggests that there is a strong association between W437R-I503K in PvDBPII in Myanmar $P$. vivax isolates, but not between $\mathrm{N} 417 \mathrm{~K}$ with either W437R or I503K.

The rate of non-synonymous mutations $(K \mathrm{n})$ and that of synonymous mutations $(K \mathrm{~s})$ is generally used as an indicator of the action of natural selection in most coding gene sequences [27]. Negative selection acting on coding genes can usually be identified when non-synonymous mutations are not advantageous, so the rate of synonymous mutations surpasses that of non-synonymous mutations $(K \mathrm{~s}$ $>K \mathrm{n}$ ). Meanwhile, positive selection is acting on a gene, when non-synonymous mutations can be beneficial (e.g. to avoid the host immune response), and the rate of nonsynonymous mutations exceed that of synonymous mutations $(K \mathrm{n}>K \mathrm{~s})$. Previous studies on PvDBPII diversity indicate that the high rate of non-synonymous mutations $(K n)$ relative to that of synonymous mutations $(K s)$ reflects positive selection pressure $[14,31,36]$. The positive value of $\mathrm{Kn} / K \mathrm{~s}$ (3.299) for all 54 sequences suggest that PvDBPII in Myanmar $P$. vivax isolates is under positive natural selection. The positive values of Tajima's D $(0.2635, \mathrm{P}>0.10)$ and Fu and Li's D $(1.867, \mathrm{P}<0.02)$ and F statistics $(1.535$, $0.1>\mathrm{P}>0.05)$ indicate that PvDBPII alleles occur at more intermediate frequencies than expected and that few alleles are rare or near fixation, which is consistent with the action of balancing selection, which maintains allelic variation in a population. These results collectively suggested that strong balancing selection, probably by host immune selection pressure, occurs at PvDBPII in the Myanmar isolates.

Polymorphism in B- and T-cell epitopes of parasite antigens may well enable parasites to escape host immune responses, as a polymorphism in the epitopes can up or down regulate $\mathrm{T}$-cell responses to the index peptide or completely arrest an immune response, assisting escape of the parasite from the host immune system [37]. The high degree of nucleotide diversity and high rates of nonsynonymous to synonymous mutations are observed in known or predicted B- and T-cell epitopes and MHC binding regions of PvDBPII in the Myanmar isolates. Overall nucleotide diversity values for these epitopes and regions were greater than those for the entire PvDBPII. In particular, high levels of nucleotide diversity were identified in peptides 48 and Ia, which is comparable to Brazilian isolates [30]. Positive Tajima's D values for these epitopes also suggested that positive natural selection preferentially acted on the epitopes in PvDBPII in the Myanmar isolates. These epitopes are predicted to be exposed to the surface of the PvDBP molecule [30]. The putative changes in protein structure may alter antibody binding efficacy of a particular epitope, thereby allowing escape from the host protective immune response [13,30].

Many factors may contribute to genetic diversity in malaria populations, including mutations, intragenic recombination, natural selection, gene flow between different regions, and population size. Although it remains controversial, it has been suggested that recombination also contributes to the diversity of PvDBPII $[30,36]$. The existence of recombination events and the decline in the LD with increasing distance between nucleotide sites suggest that in addition to natural selection meiotic recombination may also contribute to maintain the diversity of PvDBPII among Myanmar P. vivax isolates, as reported previously in Brazil, Colombia, and Sri Lanka [30,31].

\section{Conclusion}

The present study is the first report on the genetic polymorphism and natural selection of PvDBPII in Myanmar $P$. vivax isolates. Myanmar $P$. vivax isolates are genetically diverse and this seems to have arisen by a immune evasion mechanism, in which positive natural selection and 
recombination events maintained the diversity in the form of balancing selection. However, further studies using a larger number of isolates collected from different geographical regions in Myanmar will be helpful to reveal the nationwide parasite heterogeneity and the implementation of malarial control programmes in Myanmar.

\section{Acknowledgements}

We are grateful to all blood donors and the staffs at Department of Medical Research (Upper Myanmar). This work was supported by a grant of the Korea Centers for Disease Control and Prevention (2009-E54004-00) and the National Research Foundation of Korea (NRF) grant funded by the Korea government (MEST) (2011-0028135).

\section{Author details}

${ }^{1}$ Department of Parasitology and Institute of Health Sciences, Gyeongsang National University School of Medicine, Jinju 660-751, Korea. ${ }^{2}$ Department of Anatomy, Yonsei University College of Medicine, Seoul 120-752, Korea. ${ }^{3}$ Division of Malaria and Parasitic Diseases, National Institute of Health, Korea Centers for Disease Control and Prevention, Osong 122-701, Korea. ${ }^{4}$ Department of Pathology, University of Florida, J-566, 1600 SW Archer Road, Gainesville, FL 32610, USA. ${ }^{5}$ Department of Health, Vector Borne Diseases Control Project, 36 Theinbyu Road, Mandalay, Myanmar. ${ }^{6}$ Department of Internal Medicine and Inha Research Institute for Medical Sciences, Inha University School of Medicine, Incheon 400-712, Korea. ${ }^{7}$ Department of Parasitology and Inha Research Institute for Medical Sciences, Inha University School of Medicine, Incheon 400-712, Korea.

\section{Authors' contributions}

$\mathrm{HLJ}$ and JMK performed all the experiments and analysed the sequence data. SUM, JYK, HWL, KL, and BKN collected the blood samples. SUM performed sequence and phylogenetic analyses. BKN and TSK designed the study and supervised the study process. BKN wrote the paper. TSK, WMS, and JSL assisted in writing and editing the manuscript. All authors read and approved the final manuscript.

\section{Competing interests}

The authors declare that they have no competing interests.

Received: 24 October 2011 Accepted: 1 March 2012

Published: 1 March 2012

\section{References}

1. Adams JH, Sim BK, Dolan SA, Fang X, Kaslow DC, Miller LH: A family of erythrocyte binding proteins of malaria parasites. Proc Natl Acad Sci USA 1992, 89:7085-7089.

2. Barnwell JW, Nichols ME, Rubinstein P: In vitro evaluation of the role of the Duffy blood group in erythrocyte invasion by Plasmodium vivax. J Exp Med 1989, 169:1795-1802.

3. Wertheimer SP, Barnwell JW: Plasmodium vivax interaction with the human Duffy blood group glycoprotein: identification of a parasite receptor-like protein. Exp Parasitol 1989, 69:340-350

4. Horuk R, Chitnis C, Darbonne W, Colby T, Rybicki A, Hadley T, Miller L: A receptor for the malarial parasite Plasmodium vivax: the erythrocyte chemokine receptor. Science 1993, 261:1182-1184.

5. Michon PA, Arevalo-Herrera M, Fraser T, Herrera S, Adams JH: Serologic responses to recombinant Plasmodium vivax Duffy binding protein in a Colombian village. Am J Trop Med Hyg 1998, 59:597-599.

6. Xainli J, Baisor M, Kastens W, Bockarie M, Adams JH, King CL: Agedependent cellular immune responses to Plasmodium vivax Duffy binding protein in humans. J Immunol 2002, 169:3200-3207.

7. Michon P, Fraser T, Adams J: Naturally acquired and vaccine-elicited antibodies block erythrocyte cytoadherence of the Plasmodium vivax Duffy binding protein. Infect Immun 2000, 68:3164-3171.

8. Grimberg B, Udomsangpetch R, Xainli J, McHenry A, Panichakul T, Sattabongkot J, Cui L, Bockarie M, Chitnis C, Adams J, Zimmerman PA King CL: Plasmodium vivax invasion of human erythrocytes inhibited by antibodies directed against the Duffy binding protein. PLoS Med 2007, 4: e337.

9. Cerávolo I, Souza-Silva F, Fontes C, Braga E, Madureira A, Krettli A, Souza J, Brito C, Adams J, Carvalho L: Inhibitory properties of the antibody response to Plasmodium vivax Duffy binding protein in an area with unstable malaria transmission. Scand J Immunol 2008, 67:270-278.

10. Chitnis CE, Miller LH: Identification of the erythrocytes binding domains of Plasmodium vivax and Plasmodium knowlesi proteins involved in erythrocyte invasion. J Exp Med 1994, 180:497-506.

11. Chitnis CE, Chaudhuri A, Horuk R, Pogo AO, Miller LH: The domain on the Duffy blood group antigen for binding. J Exp Med 1996, 184:1531-1536.

12. Ranjan A, Chitnis CE: Mapping regions containing binding residues within functional domains of Plasmodium vivax and Plasmodium knowlesi erythrocyte-binding proteins. Proc Natl Acad Sci USA 1999, 96:14067-14072.

13. Cole-Tobian J, Cortes A, Baisor M, Kastens W, Xainli J, Bockarie M, Adams JH, King CL: Age-acquired immunity to a Plasmodium vivax invasion ligand, the Duffy binding protein. J Infect Dis 2002, 186:531-539.

14. Cole-Tobian J, King CL: Diversity and natural selection in Plasmodium vivax Duffy binding protein gene. Mol Biochem Parasitol 2003, 127:121-132.

15. Tsuboi T, Kappe SHI, Alyaman F, Prickett MD, Alpers M, Adams JH: Natural variation within the principal adhesion domain of the Plasmodium vivax Duffy binding-protein. Infect Immun 1994, 62:5581-5586.

16. Ampudia E, Patarroyo M, Patarroyo M, Murillo L: Genetic polymorphism of the Duffy receptor binding domain of Plasmodium vivax in Colombian wild isolates. Mol Biochem Parasitol 1996, 78:269-272.

17. Xainli J, Adams J, King C: The erythrocyte binding motif of Plasmodium vivax duffy binding protein is highly polymorphic and functionally conserved in isolates from Papua New Guinea. Mol Biochem Parasitol 2000, 111:253-260

18. Kho W, Chung J, Sim E, Kim D, Chung W: Analysis of polymorphic regions of Plasmodium vivax Duffy binding protein of Korean isolates. Korean J Parasitol 2001, 39:143-150.

19. Sousa T, Cerávolo I, Fernandes Fontes C, Couto A, Carvalho L, Brito C: The pattern of major polymorphisms in the Duffy binding protein ligand domain among Plasmodium vivax isolates from the Brazilian Amazon area. Mol Biochem Parasitol 2006, 146:251-254.

20. Gosi P, Khusmith S, Khalambaheti T, Lanar D, Schaecher K, Fukuda M, Miller S: Polymorphism patterns in Duffy-binding protein among Thai Plasmodium vivax isolates. Malar J 2008, 7:112.

21. Babaeekho L, Zakeri S, Djadid ND: Genetic mapping of the duffy binding protein (DBP) ligand domain of Plasmodium vivax from unstable malaria region in the middle east. AmJTrop Med Hyg 2009, 80:112-118.

22. VanBuskirk K, Sevova E, Adams J: Conserved residues in the Plasmodium vivax Duffy-binding protein ligand domain are critical for erythrocyte receptor recognition. Proc Natl Acad Sci USA 2004, 101:15754-15759.

23. VanBuskirk KM, Cole-Tobian JL, Baisor M, Sevova ES, Bockarie M, King CL, Adams $\mathrm{JH}$ : Antigenic drift in the ligand domain of Plasmodium vivax Duffy binding protein confers resistance to inhibitory antibodies. $J$ Infect Dis 2004, 190:1556-1562.

24. Moon SU, Na BK, Kang JM, Kim JY, Cho SH, Park YK, Sohn WM, Lin K, Kim TS: Genetic polymorphism and effect of natural selection at domain I of apical membrane antigen-1 (AMA-1) in Plasmodium vivax isolates from Myanmar. Acta Trop 2010, 114:71-75.

25. Tamura K, Dudley J, Nei M, Kumar S: MEGA4: Molecular Evolutionary Genetics Analysis (MEGA) software version 4.0. Mol Biol Evol 2007 24:1596-1599.

26. Librado P, Rozas J: DnaSP v5: a software for comprehensive analysis of DNA polymorphism data. Bioinformatics 2009, 25:1451-1452.

27. Nei M, Gojobori T: Simple methods for estimating the numbers of synonymous and nonsynonymous nucleotide substitutions. Mol Biol Evol 1986, 3:418-426.

28. Tajima F: Statistical method for testing the neutral mutation hypothesis by DNA polymorphism. Genetics 1989, 123:585-595.

29. Fu YX, Li WH: Statistical tests of neutrality of mutations. Genetics 1993 , 133:693-709.

30. Sousa TN, Tarazona-Santos EM, Wilson DJ, Madureira AP, Falcão PRK, Fontes CJF, Gil LHS, Ferreira MU, Carvalho LH, Brito CFA: Genetic variability and natural selection at the ligand domain of the Duffy binding protein in Brazilian Plasmodium vivax populations. Malar J 2010, 9:334. 
31. Premaratne PH, Aravinda BR, Escalante AA, Udagama PV: Genetic diversity of Plasmodium vivax Duffy Binding Protein II (PvDBPII) under unstable transmission and low intensity malaria in Sri Lanka. Infect Genet Evol 2011, 11:1327-1339.

32. Lin K: Malaria control in Myanmar. In Asian Parasitology, Malaria in Asia Edited by: Kano S, Tongol-Rivera P. The federation of Asian Parasitologists; 2005:123-134

33. Moon SU, Lee HW, Kim JY, Na BK, Cho SH, Lin K, Sohn WM, Kim TS: High frequency of genetic diversity of Plasmodium vivax field isolates in Myanmar. Acta Trop 2009, 109:30-36.

34. Kim TS, Kim HH, Lee SS, Na BK, Lin K, Cho SH, Kang YJ, Kim DK, Sohn Y, Kim H, Lee HW: Prevalence of Plasmodium vivax VK210 and VK247 subtype in Myanmar. Malar J 2010, 9:195.

35. Hans D, Pattnaik P, Bhattacharyya A, Shakri A, Yazdani S, Sharma M, Choe H, Farzan M, Chitnis C: Mapping binding residues in the Plasmodium vivax domain that binds Duffy antigen during red cell invasion. Mol Microbiol 2005, 55:1423-1434.

36. Martinez P, Suarezi CF, Cardenas PP, Patarroyo MA: Plasmodium vivax Duffy binding protein: a modular evolutionary proposal. Parasitology 2004, 128:353-366.

37. Tanabe K, Escalante A, Sakihama N, Honda M, Arisue N, Horii T, Culleton R, Hayakawa T, Hashimoto T, Longacre S, Pathirana S, Handunnetti S, Kishino H: Recent independent evolution of msp1 polymorphism in Plasmodium vivax and related simian malaria parasites. Mol Biochem Parasitol 2007, 156:74-79.

doi:10.1186/1475-2875-11-60

Cite this article as: Ju et al: Genetic polymorphism and natural selection of Duffy binding protein of Plasmodium vivax Myanmar isolates. Malaria Journal 2012 11:60.

\section{Submit your next manuscript to BioMed Central and take full advantage of:}

- Convenient online submission

- Thorough peer review

- No space constraints or color figure charges

- Immediate publication on acceptance

- Inclusion in PubMed, CAS, Scopus and Google Scholar

- Research which is freely available for redistribution

Submit your manuscript at www.biomedcentral.com/submit 